\title{
Dresde et les Wettin (1697-1756). Ascension d'une dynastie, construction d'une capitale
}

\section{Philippe Saudraix}

\section{(2) OpenEdition}

\section{Journals}

Édition électronique

URL : http://journals.openedition.org/ifha/185

DOI : 10.4000/ifha. 185

ISSN : 2198-8943

\section{Éditeur}

IFRA - Institut franco-allemand (sciences historiques et sociales)

\section{Édition imprimée}

Date de publication : 6 février 2011

ISSN : 2190-0078

\section{Référence électronique}

Philippe Saudraix, «Dresde et les Wettin (1697-1756). Ascension d'une dynastie, construction d'une capitale », Revue de l'IFHA [En ligne], 3 | 2011, mis en ligne le 19 avril 2012, consulté le 03 mai 2019.

URL : http://journals.openedition.org/ifha/185 ; DOI : 10.4000/ifha.185

Ce document a été généré automatiquement le 3 mai 2019.

(CIFHA 


\title{
Dresde et les Wettin (1697-1756). Ascension d'une dynastie, construction d'une capitale
}

\author{
Philippe Saudraix
}

\section{NOTE DE L'ÉDITEUR}

Philippe Saudraix a soutenu sa thèse de doctorat sous la direction d'olivier Chaline le 5 mars 2011, à l'université Paris IV-Sorbonne. Au cours de la préparation de sa thèse, il a été lauréat de la « bourse Mandrou » en 2006 et bénéficiaire de plusieurs bourses de courte durée accordées par la MHFA.

À l'été 1697, l'électeur de Saxe Auguste le Fort abjure le luthéranisme et devient roi de Pologne. Cette union personnelle introduit les Wettin albertins dans le rang des familles souveraines d'Europe, et leur permet de jouer un important rôle politique, comme le montrent la guerre du Nord (1700-1721), la guerre de Succession d'Autriche (1740-1748) et la guerre de Sept Ans (1756-1763). Par ailleurs, en devenant catholique et royale, cette famille électorale peut offrir un avenir prestigieux à sa descendance. L'unique fils légitime d'Auguste le Fort, Frédéric-Auguste II, épouse une nièce de Charles VI, MarieJosèphe de Habsbourg. À la génération suivante, Marie-Amélie épouse le futur Charles III d'Espagne, Marie-Josèphe le Dauphin, tandis que Clément-Venceslas devient archevêque de Trèves, et Albert vice-roi de Hongrie. Cette ascension fulgurante aurait pu être durable, n'était la violence avec laquelle Frédéric II frappa la Saxe en 1756: la ruine de l'électorat et le changement radical de politique en 1763 mirent un coup d'arrêt à cet essor'.

Or, dans le même temps, Dresde, ville-résidence de l'électeur de Saxe, connaît une croissance inouïe : en soixante ans, sa population triple, tandis que l'espace urbain se densifie et se dilate tout à la fois. En outre, la ville se transforme : elle est conquise par 
l'art baroque, tout en devenant propre, sûre et agréable. La Dresde qui est entrée dans le patrimoine culturel européen date de la période des rois saxons de Pologne, avec les jardins du Zwinger, la Frauenkirche, la Hofkirche et la terrasse Brühl. Cet essor urbain est brutalement interrompu lors de la guerre de Sept Ans².

Ce constat du parallélisme des destinées d'une dynastie et de sa ville-résidence intrigue : en quoi l'essor des Wettin albertins et les transformations de Dresde sont-ils liés? Aussi évidente qu'elle puisse paraître, cette question n'a jamais été traitée, malgré l'hypothèse avancée par le premier historien de Dresde, Otto Ludwig Richter : «Une époque encore plus brillante s'ouvrit lorsque, avec l'acquisition de la couronne de Pologne par Auguste le Fort, Dresde devint le centre d'une politique de grande puissance à l'échelle européenne. La cour royale s'entoura d'une splendeur inouïe, son siège devait être transformé en un cadre digne du déploiement de la magnificence royale ${ }^{3}$." Cette phrase mérite un développement qui permette de comprendre comment et pourquoi la royauté saxonne de Pologne a pu entraîner l'essor de Dresde. Mais pour des raisons à la fois disciplinaires et idéologiques, ce sont deux historiographies distinctes qui se sont développées : Dresde a surtout attiré les historiens de l'art, mais guère les spécialistes des villes et des sociétés urbaines ; les Wettin, quant à eux, ont été durablement mal jugés, tenus pour des esthètes responsables de la médiocrité de l'État saxon et de la disparition politique de la Pologne au XIXe siècle.

Pourtant, le parallélisme de l'ascension des Wettin et de la transformation de Dresde n'est pas seulement d'ordre régional, mais il concerne toute l'histoire du Saint-Empire. Il est connu qu'à l'exception du port de Hambourg, les villes-résidences sont au XVIIIe siècle les villes les plus dynamiques de l'espace germanique ${ }^{4}$. L'étude d'un cas précis doit permettre de mieux connaître le phénomène de la ville-résidence, en faisant se rencontrer l'histoire politico-dynastique et l'histoire urbaine. Cela nécessite de prendre en compte une histoire complexe qui, dans son espace et sa temporalité, se joue à plusieurs niveaux complémentaires : celui de la ville au sens juridique du terme, corps politique autonome représenté par le magistrat et très attaché à la confession luthérienne ; celui de l'espace urbain dont une partie est sous la juridiction immédiate de l'électeur ; celui de la capitale politique, administrative et religieuse de la Saxe électorale; celui de la ville-résidence d'un roi catholique et de sa famille ; celui, enfin, d'une capitale culturelle de dimension européenne. Cette complexité de nature exclut de se restreindre à un seul type de sources: il est nécessaire de croiser les archives communales, les archives de l'État de Saxe (non seulement le conseil privé et le cabinet privé, mais aussi le bailliage et le gouvernement militaire), les imprimés (récits de voyage, encyclopédies, panégyriques), les plans, les tableaux de Bellotto, les bâtiments, même ceux reconstitués après le bombardement de 1945.

De ce point de vue, étudier les liens qui unissent une dynastie et sa ville-résidence, c'est certes considérer les bâtiments réalisés par la première, ainsi que son œuvre d'urbanisme, son mécénat et ses fêtes. Mais ne jamais quitter cette perspective conduirait à une vision partiale de la réalité, faisant fi des acteurs non dynastiques. Plutôt que de postuler l'absolutisme des Wettin et l'hégémonie de la cour de Saxe, mieux vaut partir de la ville prise dans son ensemble, afin d'y discerner le rôle qu'a joué la dynastie dans ses destinées. Avoir adopté ce principe nous a conduits à examiner dans un premier temps le cadre de la transformation de Dresde, les aspects institutionnels, les commanditaires et les conditions matérielles; dans un second temps vient l'inventaire de toutes les transformations urbaines, ce qui a été bâti soit par la dynastie, soit par le magistrat, soit 
par les commanditaires privés, sans oublier les espaces non habités (rues, fortifications, jardins, cimetières, etc.) ; c'est seulement ensuite que peut être examinée la question des fonctions de la ville-résidence, qui est à la fois capitale politique et administrative de la Saxe électorale et du roi de Pologne, mais aussi capitale économique et commerciale, enfin capitale culturelle et religieuse.

Trois conclusions essentielles peuvent être énoncées. Tout d'abord, les Wettin albertins expriment l'identité de leur famille dans leur ville-résidence, dans les bâtiments qu'ils édifient, dans les collections qu'ils rassemblent et dans les fêtes qu'ils organisent. Trois éléments sont décisifs. Les Wettin insistent avant tout sur le prestigieux passé de leur famille, depuis leur parent mythologique, Hercule, jusqu'au fondateur de la lignée électorale albertine, Maurice. Cet aspect trouve son prolongement dans la splendeur et l'abondance du présent : la royauté saxonne de Pologne est présentée comme le retour de l'âge d'or, ce dont témoignent le palais Japonais, les pavillons du Zwinger et les multiples collections dynastiques. Enfin, la piété catholique devient un élément essentiel de l'identité dynastique, surtout sous Frédéric-Auguste II et son épouse Marie-Josèphe de Habsbourg, à qui Dresde doit d'être devenue une capitale catholique.

Toutefois, jamais ce qui a été réalisé dans l'ensemble de la ville de Dresde n'aurait pu l'être sans la participation d'un grand nombre de commanditaires non dynastiques, que ce soit le magistrat, les nobles ou de simples particuliers. Les Wettin ne sont certes pas étrangers à cette fièvre bâtisseuse, en ce qu'ils sont la première cause de l'enrichissement des habitants de la ville-résidence : la présence de la dynastie, le développement de sa cour et de son administration sont la base de la prospérité de Dresde, par les emplois qui dépendent directement ou indirectement de la construction et de l'approvisionnement de la ville, sans oublier les bourgeois qui s'enrichissent par la spéculation immobilière ou par la location de logements à tous ceux qui ne peuvent être propriétaires dans Dresde 5 . Pour cette raison, il est impossible de ne voir en cette ville qu'une capitale culturelle, voire une annexe de la cour des Wettin. Dresde est également une capitale administrative et militaire, ainsi qu'un foyer économique et commercial. Bien plus, en raison du privilège de l'autonomie communale, l'électeur de Saxe ne peut agir indépendamment du magistrat, dont la vigueur est indéniable. L'étude des transformations de Dresde met en jeu la question des relations entre les pouvoirs communaux et électoraux, comme l'attestent les conflits autour des aménagements des trois places de marché de la ville. L'identité communale doit d'autant moins être sous-estimée que dans le cas de Dresde, elle s'est doublée d'un conflit religieux : contre l'électeur catholique, le magistrat fait de la ville-résidence une capitale luthérienne, dont la meilleure expression est la Frauenkirche, l'un des plus grands chantiers communaux du XVIIIe siècle.

Pour autant, il est faux de considérer que dans la ville-résidence se juxtaposent deux espaces socioculturels, celui de la cour et celui de la bourgeoisie ${ }^{6}$. D'une part, ce serait oublier que les bourgeois tirent profit et fierté de la proximité des Wettin. D'autre part, c'est l'ensemble de l'espace urbain qui est aménagé par la dynastie, par l'intermédiaire du gouvernement de la place forte et de l'administration civile et militaire des bâtiments, deux institutions qui jouent un rôle considérable dans l'assainissement et dans l'embellissement urbain. Ainsi, l'investissement dynastique se manifeste dans la totalité de la ville-résidence. Cela vient de ce que tout l'espace urbain est à la fois ville et résidence ${ }^{7}$. Il y a ainsi une mise en image de la dynastie dans la ville : à l'ancienneté, la splendeur et la piété de la dynastie répondent celles de la ville. La ville-résidence est tout entière un miroir du prince, ce qui permet d'éclairer le sens de l'embellissement urbain : 
à la beauté intérieure du prince répond la beauté visible de la ville-résidence ou, pour le dire autrement, Dresde est l'écrin dans lequel prend place le joyau du pays, l'électeur sacré roi. Or, un prince du Saint-Empire pieux et glorieux peut espérer la dignité impériale et la félicité éternelle. Il n'est alors pas étonnant d'observer quel est l'unique modèle de la transformation de Dresde - éventuellement par la médiation de Vienne et, dans une moindre mesure, de Paris: Rome, capitale impériale et sacrée. Le plus grand moteur de la transformation de Dresde est le désir de gloire des Wettin, gloire à la fois temporelle et spirituelle, dont la ville-résidence est le miroir.

\section{NOTES}

1. Pour l'histoire de la Saxe, Reiner Groß, Geschichte Sachsens, Berlin : Edition Leipzig, 2001.

2. Pour l'histoire de Dresde, Reiner Groß, Uwe John (dir.), Geschichte der Stadt Dresden, t. II, Stuttgart : Theiss, 2006.

3. Otto Ludwig Richter, Geschichte der Stadt Dresden in den Jahren 1871 bis 1902, Dresden : Zahn \& Jaensch, 1903, p. 2 : « Aber noch glänzendere Zeiten kamen, als mit der Erwerbung der Krone Polens durch August den Starken Dresden ein Ausgangspunkt europäischer Großmachtpolitik wurde. Mit unerhörtem Glanze umgab sich der neue Königshof, sein Wohnsitz sollte zu einem würdigen Rahmen königlicher Prachtentfaltung umgestaltet werden. »

4. Étienne François, « Des républiques marchandes aux capitales politiques. Remarques sur la hiérarchie urbaine du Saint-Empire à l'époque moderne » in : Revue d'histoire moderne et contemporaine, 25, 1978, p. 587-603.

5. Le terme «bourgeois » est ici pris dans son seul sens juridique, qui est de loin le plus courant dans les sources que nous avons dépouillées. Tant que l'on n'aura pas prouvé que les sens juridique, économique, social et culturel de ce mot recouvrent exactement la même réalité humaine, la logique interdit de mener tout raisonnement postulant qu'un bourgeois au sens juridique l'est également aux sens économique, social et culturel.

6. Le cas est analogue à celui de Vienne. Voir John Philip Spielman, The City and the Crown : Vienna and the Imperial Court 1600-1740, West Lafayette (Ind.) : Purdue Univ. Press, 1993.

7. Jakob \& Wilhelm Grimm, Deutsches Wörterbuch, Leipzig : S. Hirzel, 1854 : « Residenz : für Ort, besonders Stadt, in welcher der Landesfürst seinen dauernden Aufenthalt hat »; « Residenzstadt : für eine Stadt, welche Residenz ist ». 